\title{
Research into Network Optimization based on PSO Algorithm in RFID Network Layout
}

\author{
Jiangang Jin \\ Software Technology Vocational College, North China University of Water \\ Resources and Electric Power, Zhengzhou 450045, China \\ henanjingjg@sina.com
}

\begin{abstract}
How to make the RFID reader have better coverage and improve the accuracy of transmitting information has always been the research direction. In this paper, the RFID reader individual is corresponded with particles in PSO. First of all, establish the optimization function by interfering with signals in the RFID network with distribution density of reader and data transmission load as the target of optimization. Second, aiming at the deficiency of PSO algorithm the Monte Carlo simulation and opposition-based learning strategy are introduced to make particles of the PSO algorithm jump out of the local optimization and improve the algorithm's efficiency. Finally, the simulation experiment shows that algorithm in this paper and QPSO have been significantly improved in signal interference, reader distribution density and data transmission load, so they can effectively improve the efficiency of RFID network layout.
\end{abstract}

Keywords : RFID coverage; opposition-based learning strategy

\section{Introduction}

Radio Frequency Identification (RFID) has provided rapid and universal data collection and transmission scheme for many industries, so it has broad application prospect. The deployment of RFID network is one of the main direction of the study of the Internet of things under the RFID technology and reasonable network deployment structure to maximize the RFID network monitoring capabilities [1]. Domestic and foreign scholars for RFID network deployment were studied. Literature [2] proposed a grid based and coverage model of RFID reader deployment algorithm. The algorithm first introduced the reader coverage model, and then by meshing algorithm for the calculation of the specific location of each grid and the specific location of the obstacle, finally by RFID reader deployment algorithm to calculate the reader to deploy them to the best position. Experimental results show that this algorithm improve the RFID reader deployment efficiency has important significance; literature [3] is proposed based on a tabu search algorithm for the reader deployment method. Increase in the read and write device capacity constraint reader deployment model based on and using tabu search algorithm to find the optimal deployment. The performance of the algorithm is stable through simulation. And the comparison of the data of the genetic algorithm based on Tabu Search reader deployment method in coverage was greater than the genetic algorithm at the same time, the number of iterations is less than genetic algorithm; Literature [4] proposed a Lozi map with contraction factor and inertia weight of the particle swarm algorithm based on. In there to read and write crossover cover and label interference factors in complex communication environment, to find the best position of reader, contraction factor and inertia weight ensures the accuracy of algorithm preliminary search speed and search in the late phase of. Simulation results show that the guarantee coverage rate and maximum cross interference level constrained minimum conditions, the algorithm is better than the basic PSO algorithm and based on Logistic mapping PSO 
algorithm has less iterative times, fitness is better, more superiority; Literature [5] is proposed based on grid partition mechanism of static RFID reader deployment method, the two-dimensional and three-dimensional structure in reader deployment. And a distance vector failure warning algorithm for RFID reader failure in the network is proposed. The algorithm is simple, efficient and short of deployment cycle, and the warning algorithm is fast and accurate. Literature [6] proposes an integrated RFID reader - wireless sensor network node deployment optimization strategy. Through an iterative, find the optimal coordinate position of the intelligent node, compared to the particle swarm algorithm, improved particle swarm algorithm to accelerate the search for optimal node speed, and can quickly and effectively converge to the optimal solution. Literature [7] proposed a matrix quantization strategy, the reader electromagnetic radiation model and coverage area are mapped and coverage area three-dimensional matrix for reader three-dimensional matrix. This method can reflect more detailed and realistic RFID reader in a variety of application scenarios of the deployment.

In order to better cover for RFID reader and control, the usual practice is to use a large number of reader-reader in the monitored area in order to achieve complete coverage of the theoretical disadvantage is that there is signal interference between the RFID reader, caused by the large number of costs, network load imbalance and other issues. Paper establishes RFID signal interference on the basis of the above disadvantages, the reader density and data transfer load three parameters as the optimization target, establish RFID network deployment model multi-objective algorithm is further optimized by PSO, while the introduction of a reverse learning strategies so that the PSO algorithm to escape from local optimum particle, improve the efficiency of the algorithm. The PSO improved algorithm applied to the RFID network to deploy multi-objective optimization model is solved, simulation experiments show that our algorithm can effectively improve the efficiency of the RFID network deployments.

\section{RFID Network Coverage Model}

In a real environment, RFID tags distributed network deployment need to consider factors density, RFID reader costs and other additional economic cost and other issues, this article from signal interference, reader density and data transfer load three aspects to consider, multi-objective RFID network coverage model, set to cover the target as follows:

$$
F=\omega_{1} f_{1}+\omega_{2} f_{2}+\omega_{3} f_{3}
$$

In the formula, $\omega_{1}, \omega_{2}, \omega_{3}$ refer to the weight value of their respective targets, $f_{1}$ refers to the signal interference value, $f_{2}$ refers to the density of the distribution density, and $f_{3}$ refers to the data transmission size. This paper mainly discusses how to minimize the coverage target value, which is also the direction of researching RFID network coverage model, that is $\min F$.

\section{RFID Network Coverage based on PSO Algorithm and Opposition-based Learning}

\subsection{Particle Swarm Optimization and RFID Network Coverage}

Make corresponding coverage target value of individuals in RFID reader correspond with individual particles in PSO algorithm. Through the optimal position of particles in algorithm in this paper, i.e. the optimal position of RFID reader individuals, $\min F_{i} \rightarrow i_{\text {best }}$. 


\subsection{PSO Algorithm based on Monte Carlo}

Particle Swarm Optimization (PSO) is an algorithm simulating the foraging behaviors of bird population in flight; suppose in the $N$ dimensional search space, the location and speed of the $i$ particle are respectively $Z_{i}=\left(z_{i 1}, z_{i 2}, \cdots, z_{i N}\right)$ and $V_{i}=\left(v_{i 1}, v_{i 2}, \cdots, v_{i N}\right)$. $P_{i}=\left(p_{i 1}, p_{i 2}, \cdots, p_{i N}\right)$ refers to the optimal position of the $i$ particle in flight, $P_{g}=\left(p_{g 1}, p_{i 2}, \cdots, p_{i N}\right)$ refers to the optimal position of the population, and the particles update the speed and position according to the following formula:

$$
\begin{aligned}
v_{i n}(t+1)= & \omega \times v_{i n}(t)+c_{1} \times \operatorname{rand}() * \\
& \left(p_{i n}(t)-z_{\text {in }}(t)\right)+c_{2} \times \operatorname{rand}()^{*}\left(p_{g n}(t)-z_{\text {in }}(t)\right) \\
z_{\text {in }}(t+1) & =z_{\text {in }}(t)+v_{\text {in }}(t+1)
\end{aligned}
$$

Herein, $t$ refers to iteration; $c_{1}$ and $c_{2}$ refer to learning factors; rand () is a random number between $[0,1]$; and $\omega$ is a inertia weight.

In this paper, through the Monte Carlo simulation, the expression of particle swarm individual evolves as follows:

$$
z_{\text {in }}(t+1)=v_{\text {in }}(t) \pm \frac{L_{\text {in }}(t)}{2} \ln \left(\frac{1}{u_{\text {in }}(t)}\right)
$$

Herein, set $u_{i d}(t) \rightarrow U(0,1)$, which is mainly a random number distributing in $(0,1)$. $L(t)$ determines the performance of particle swarm algorithm. Introduce the global variance Mbest and suppose relationship of the optimal position of individual particles is as follows:

$$
\operatorname{Mbest}(t)=\left(\frac{1}{N} \sum_{M}^{1} p_{i 1}(t), \frac{1}{N} \sum_{M}^{1} p_{i 2}(t), \cdots \frac{1}{N} \sum_{M}^{1} p_{i D}(t)\right)
$$

The difference equation (4) in the time $t$ evolution of the position $z_{\text {in }}(t)$ of the body $\operatorname{Mbest}(t)$ and the equation (5) in between positions as evaluation of $L(t)$.

$$
z_{\text {in }}(t+1)=v_{\text {in }}(t) \pm \lambda \mid z_{\text {in }}(t)-\text { Mbest }_{\text {in }}(t) \mid \ln \left(\frac{1}{u_{\text {in }}(t)}\right)
$$

In formula (6), $\lambda$ refers to the disturbance factor, which will directly affect the factor's convergence. In this paper, it can be found through experiments that when $\lambda$ is larger than 1.812, the algorithm shows a divergence trend; otherwise, it shows a convergence trend. Therefore, this paper chooses the value of $\lambda$ as 1.672, which can guarantee that PSO has good convergence, which reduces the operation time.

\subsection{Opposition-based Learning Strategy}

Opposition-based learning (OBL) is a strategy to choose a better solution from the current solution and the opposition point as individuals of the next generation. With rapid speed, good optimization capacity and fitness, this strategy can effectively improve diversity of population so as to avoid the algorithm to fall into local optimization.

Definition 1: opposition point. Suppose $X=\left(x_{1}, x_{2}, \cdots, x_{D}\right)$ is a point in the $D$ dimensional space, and $x_{1}, x_{2}, \cdots, x_{D} \in R, x_{j}=\left[a_{j}, b_{j}\right]$, the opposition point is $\hat{X}=\left(\hat{x}_{1}, \hat{x}_{2}, \cdots, \hat{x}_{D}\right)$, so the definition is as follows:

$$
\widehat{X}_{j}=a_{j}+b_{j}-X_{j}
$$

Definition 2: opposition-based optimization: suppose $X=\left(x_{1}, x_{2}, \cdots, x_{D}\right)$ is a point in 
the $D$ dimensional space, and set $f(*)$ is the fitness value function, then compare the opposition-based solution with the function's fitness value. If $f(\hat{X}) \leq f(X)$, replace $\hat{X}$ with $X$.

Definition 3: elite opposition-based solution: suppose $X=\left(x_{1}, x_{2}, \cdots, x_{D}\right)$ is a point in the $D$ dimensional space, and the extremum of the space is $X_{i}^{e}=\left(x_{1}^{e}, x_{2}^{e}, \cdots, x_{D}^{e}\right)$, or the opposition-based solution is $\widehat{X}_{i}^{e}=\left(\hat{x}_{1}^{e}, \hat{x}_{2}^{e}, \cdots, \hat{x}_{D}^{e}\right)$. The relationship between the two is as follows:

$$
\widehat{X}_{i}^{e}=\lambda\left(a_{j}+b_{j}\right)-X_{i}^{e}
$$

In the formula, $X_{i}^{e} \in\left[a_{j}, b_{j}\right], \lambda$ refers to the search space, so $\lambda a_{j}$ refers to $\min \left(x_{\lambda}\right)$ and $\lambda b_{j}$ refers to $\max \left(x_{\lambda}\right)$. Herein, the value of $\lambda a_{j}$ and $\lambda b_{j}$ changes with the constant change of the search space. When there is less search space, the range of opposition-based solution is also getting smaller. When the opposition-based solution is not within $\left[a_{j}, b_{j}\right]$, replace it by a random number produced in the border:

$$
\hat{X}_{\lambda}^{e}=\operatorname{rand}\left(a_{j}, b_{j}\right)
$$

\subsection{Optimal Judgment of Local Algorithm}

In order to further improve the convergence accuracy of PSO algorithm, thus paper adopts the variance of subgroup to measure whether the algorithm will fall into the local optimization. Set the evaluation function as $f_{1}$, and the average value of fitness is $f_{\text {aver }}$, the $\sigma$ refers to the fitness variance value, so the population fitness value is:

$$
\sigma=\sqrt{\sum_{i=1}^{n}\left|\frac{f_{1}-f_{\text {aver }}}{f}\right|}
$$

The status of the PSO algorithm is determined by $\sigma$, and if the $\sigma$ value gradually decrease, this algorithm tends to converge. Otherwise, the population is at a status of random search, unable to reach the algorithm's convergence value and the particle swarm algorithm falls into the local optimization, losing the diversity and falling into the premature convergence status. Therefore, set $\sigma_{\min }$, and when $\sigma<\sigma_{\min }$, the population falls into local optimization and needs to be processed.

\subsection{Algorithm Steps}

Step 1: determine the work area of RFID reader and the corresponding distribution of labels;

Step 2: Correspond the coverage value of RFID reader with individuals in particle swarm;

Step 3: determine the population size and iterations of the algorithm and use the Monte Carlo to determine the individual particle value.

Step 4: calculate the local convergence function value and evaluate the individual quality;

Step 5: population evolution. In case of premature convergence, opposition-learning is introduced, otherwise, implement step 6;

Step 6: after the iterations have been reached, find the RFID network coverage value corresponding to the optimal particle. 


\section{Simulation Experiment}

In this paper, the $30 \mathrm{~m} \times 30 \mathrm{~m}$ square is selected as RFID reader's work area with 30 RFID labels distributed at random. Form the RFID network through 10 RFID readers and then collect data of labels within this work area.. Herein, "*” refers to the reader. In order to show that algorithm in this paper is superior, it is compared with the QPSO algorithm.

\subsection{Comparison of Coverage Optimization}

In terms of the coverage effect in Fig.1-2, algorithm in this paper has superior coverage effect than QPSO algorithm, especially there is basically no overlapping areas in the coverage region. On the contrary, there is a large coverage area in QPSO algorithm, thus it is easy for signals to influence each other, causing conflicts and constraints.

\subsection{Comparison of 3 Constraint Indexes}

Compare algorithm in this paper with QPSO algorithm in three indexes, and it can be found from Table 1 that algorithm in this paper is superior to QPSO algorithm, which is mainly because the PSO algorithm adopts the Monte Carlo and when it is going to fall into the local optimization, opposition-based learning strategy is adopted to improve the algorithm's accuracy. It can be found from Fig.3 that algorithm in this paper has superior fitness value than QPSO algorithm, indicating that algorithm in this paper has good fitness.

Table 1. Comparison of Three Constraint Indexes

\begin{tabular}{cccc}
\hline \multirow{2}{*}{ Indicator } & Value & QPSO & $\begin{array}{c}\text { Algorithm in this } \\
\text { paper }\end{array}$ \\
& & 123.921 & 103.723 \\
Signal Strength & the optimal value & 221.035 & 180.214 \\
& the poorest value & 34.825 & 32.836 \\
& variance & 7.219 & 6.172 \\
Distribution Density & the optimal value & 9.729 & 8.691 \\
& the poorest value & 4.621 & 4.292 \\
& variance & 93.218 & 85.261 \\
Network Load & the optimal value & 187.271 & 140.06 \\
& the poorest value & 58.62 & 47.29 \\
\hline
\end{tabular}




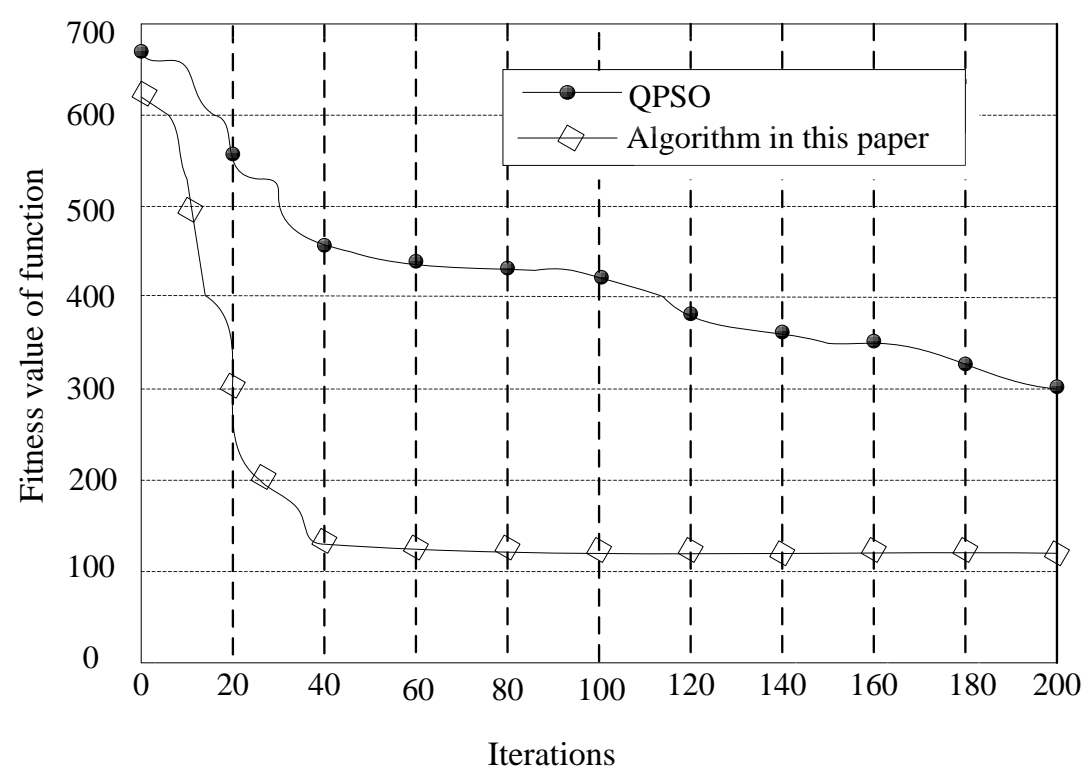

Figure 3. Comparison of the Two Algorithms' Fitness

\section{Conclusion}

This paper first establishes three parameters: signal interference of the RFID reader, reader distribution density and data transmission load as the optimization targets and conducts further optimization through the PSO algorithm. Then, the opposition-based learning strategy is introduced to reduce the possibility for particles in PSO algorithm in fall into local optimization and increase the algorithm's efficiency. Simulation experiments show that algorithm in this paper can effectively improve the efficiency of RFID network layout.

\section{References}

[1] L. X. Peng, "Key Technology for Design of RFID Simulation Deployment Platform in Manufacturing", Journal of system simulation, vol. 22, no. 3, (2010), pp. 601-605.

[2] T. X. Hong, "Research on deployment algorithm of RFID readers based on grid and coverage model", Application Research of Computers, vol. 32, no. 10, (2015), pp. 1230-1234

[3] W. Y. Hua, "RFID networks planning based on tabu search algorithms", .APPLICATION RESEARCH OF COMPUTERS, vo. 28, no. 6, (2011), pp. 2016-2019.

[4] J. Z. Cheng, "Optimal Deployment in RFID Network Based on LZM-WKPSO Algorithm", Control Engineering of China, vol. 21, no. 3, (2014), pp. 343-346.

[5] H. X. Nuo, "Research on RFID reader failure warning algorithm base on grid deployment", Bulletin of Science and Technology, vol. 30, no. 11, (2014), pp. 126-123.

[6] Z. Xiang, "Optimal Deployment of Nodes in the Integration of RFID and WSN", Wu Xi: Jiang Nan University, (2012)b.

[7] L. Yun, "Research on the Large-scale RFID reader deployment in the Three-Dimensional space", Cheng dou: University of Electronic Science and Technology of China, (2013).

\section{Authors}

Jiangang Jin, Lecturer, Master, Research Orientation: Computing Network 